\title{
EVALUATION OF SCATTERING OPERATORS FOR SEMI-INFINITE PERIODIC ARRAYS*
}

\author{
MATTHIAS EHRHARDT ${ }^{\dagger}$, JIGUANG SUN ${ }^{\ddagger}$, AND CHUNXIONG ZHENG ${ }^{\S}$
}

\begin{abstract}
Periodic arrays are structures consisting of geometrically identical subdomains, usually named periodic cells. In this paper, by taking the Helmholtz equation as a model, we consider the definition and evaluation of scattering operators for general semi-infinite periodic arrays. The well-posedness of the Helmholtz equation is established via the limiting absorption principle. A method based on the doubling procedure and extrapolation technique is first proposed to compute the scattering operators of Sommerfeld-to-Sommerfeld type. The advantages of this method are the robustness and simplicity of implementation. However, it suffers from the heavy computational cost and the resonance wavenumbers. To overcome these shortcomings, we propose another more efficient method based on a conjecture about the asymptotic behavior of limiting absorption principle solutions. Numerical evidences suggest that this method presents the same results as the first one.
\end{abstract}

Key words. Periodic arrays, Helmholtz equation, Sommerfeld-to-Sommerfeld scattering operator, dispersion diagram.

AMS subject classifications. 35B27, 65M99, 35Q60, 35J05.

\section{Introduction}

Periodic structure problems arise frequently in many up-to-date application areas like photonic crystals (PC) $[9,12,16]$, semiconductor nanostructures (e.g. quantum dots and nanocrystals), semiconductor superlattices [1, 23], metamaterials [17], or Bragg gratings of surface plasmon polariton (SPP) waveguides $[6,18]$. One of the most interesting properties of periodic structures, especially in optical applications with nano- and micro-technology, is their capability of selecting waves in a range of frequencies that are allowed to pass or are blocked through the media. Waves in periodic arrays only exist when their wavenumbers lie inside some allowed continuous bands separated by forbidden gaps. This fact corresponds mathematically to the dispersion diagram of suitable differential operator having so-called pass bands and stop bands. Since the governing wave equation is either of periodic variable coefficients, or defined on a domain consisting of periodic subregions, theoretical analysis is very limited, and numerical simulation is a fundamental tool for the design, analysis, and finally optimization of the periodic arrays.

To simulate wave propagation in periodic arrays, in many cases one needs to solve some kind of periodic PDEs on very large or even unbounded domains. The periodicity may appear in the geometry of definition domain or in the equation coefficients. For example, the determination of defect modes in photonic crystals generally necessitates solving a PDE eigenvalue problem posed on an unbounded domain with periodic medium structure [5]. For solving these PDEs numerically, a common practice is to confine the real computational domain by introducing artificial boundaries to separate a small neighborhood of the region with physical interest from its exterior domain.

*Received: September 9, 2008; accepted (in revised version): February 1, 2009. Communicated by Shi Jin.

${ }^{\dagger}$ Weierstraß-Institut für Angewandte Analysis und Stochastik, Mohrenstr. 39, 10117 Berlin, Germany (ehrhardt@wias-berlin.de). http://www.wias-berlin.de/people/ehrhardt/

${ }^{\ddagger}$ Department of Applied Mathematics and Theoretical Physics, Delaware State University, Dover, DE 19901, USA (jsun@desu.edu). http://amrc.desu.edu/web/jsun/

$\S$ Department of Mathematical Sciences, Tsinghua University, Beijing 100084, P.R. China (czheng@math.tsinghua.edu.cn). http://faculty.math.tsinghua.edu.cn/ 〜 czheng/ 
This treatment is especially beneficial if the exterior domain consists of a large number of periodic cells.

To make the PDE complete on the confined computational domain, suitable boundary conditions should be applied at artificial boundaries. For wave-like equations, the ideal boundary conditions should not only result in well-posed problems, but also mimic the perfect transportation of waves which leave the computational domain through the artificial boundaries. Taking the exterior domain as a scatterer, these boundary conditions can be also considered as some kind of scattering operators. The evaluation of these scattering operators is thus very crucial for an efficient simulation of waves in the large or unbounded periodic structures.

For the one-dimensional Schrödinger equation with cosinusoidal potential, Zheng [25] presented an analytical expression for the scattering operator in the form of a Dirichlet-to-Neumann map. This expression was then extended to more general second-order ODE problems by Ehrhardt and Zheng [2] when the coefficient functions are all symmetric. For high-dimensional periodic array problems, in general it is unreasonable to expect analytical scattering operators of any form. In [10], Joly, Li, and Fliss considered this issue by taking the periodic Helmholz equation as an example. For the damped Helmholtz equation, they proposed a modified Newton's method for evaluating the scattering operator. For the undamped equation, they resorted to the limiting absorption principle (LABP), and defined the scattering operator as the limit of those for the damped equation when the absorption coefficient goes to zero. After that, two methods were proposed. One is based on a limiting procedure: first compute the scattering operator for the damped equation, then send the absorption parameter to zero. The other is based on the modal expansion. The crucial point is to identify the traveling Bloch waves which are compatible with the LABP. Under a strict assumption that any Floquet multiplier with modulus 1 is simple, the authors of [10] presented an identifying criteria, which was further proved to be equivalent to the energy criteria [15]. In [4] Fliss and Joly further extended the idea of [10] to solve bi-periodic structure problems.

Ehrhardt, Han and Zheng [3] also considered the scattering operators for the damped Helmholz's equation in semi-infinite periodic arrays. A fast evaluation method based on the doubling technique [24] was proposed. Compared with the modified Newton's method of [10], this method is more stable and easier to understand. It was also reported that when the wavenumber lies in the stop bands of periodic arrays, this method could also present the exact scattering operators. Very recently, Sun and Zheng [20] employed the method of [3] to deal with diffraction gratings with local defects.

In this paper, we will continue the discussions in [10] and [3], and focus on the case when the wavenumber is in the pass bands. As in [10], we resort to the LABP to define the admissible solutions of Helmholz's equation. An extrapolation method combining the doubling technique with the limiting procedure is proposed. This method is robust and easy to implement. However, it suffers from heavy computational cost and the resonance wavenumbers. Considering these points, we study the asymptotic behavior of the LABP solutions. A criteria more strict than the energy one [15] to identify the LABP traveling Bloch waves is conjectured. An asymptotic method based on this conjecture is then given. In comparison with the extrapolation method, the price to pay is to solve a generalized eigenvalue problem, but the overall computational cost is greatly reduced. The numerical tests given at the end of this paper suggest that these two methods bring the same scattering operators for semi-infinite periodic arrays. 


\section{A model problem}

We consider a closed waveguide consisting of an infinite number of identical cells; see figure 2.1. There $\mathrm{C}_{j}$ denotes the $j$-th periodic cell, and $\Gamma_{j}$ the $j$-th cell boundary. The governing wave equation is the Helmholtz equation

$$
\Delta u+k^{2} n^{2} u=0,(x, y) \in \Omega=\cup_{j=0}^{+\infty} \mathrm{C}_{j},
$$

where $k$ is the reference wavenumber and $n=n(x, y)$ is the refractive index function. On each cell boundary $\Gamma_{j}$ we define two Sommerfeld data associated with the wave function $u$ as

$$
f_{j}(u)=\left.\left(\partial_{x}+i k\right) u\right|_{\Gamma_{j}}, \quad g_{j}(u)=\left.\left(\partial_{x}-i k\right) u\right|_{\Gamma_{j}},
$$

where $i$ is the imaginary unit. To clarify the physical meaning of these two data, let us return to the one-dimensional constant coefficient Helmholtz equation

$$
u_{x x}+k^{2} u=0 .
$$

Two linearly independent solutions are $e^{ \pm i k x}$. By convention, $e^{i k x}$ represents a wave traveling to the right, and $e^{-i k x}$ to the left. A simple computation leads to

$$
\left(\partial_{x}+i k\right) e^{i k x}=2 i k e^{i k x},\left(\partial_{x}-i k\right) e^{i k x}=0,
$$

and

$$
\left(\partial_{x}+i k\right) e^{-i k x}=0,\left(\partial_{x}-i k\right) e^{-i k x}=-2 i k e^{-i k x} .
$$

These expressions imply that the operator $\partial_{x}+i k$ eliminates the left-going wave while the operator $\partial_{x}-i k$ eliminates the right-going wave. Thus the functions $f_{j}$ and $g_{j}$ in (2.2) contain some information about the right-going and left-going waves respectively. They could be further regarded as incoming or outgoing relying on the location of $\Gamma_{j}$ with respect to (w.r.t.) the concerned part of domain. For example, w.r.t. $\mathrm{C}_{j}, f_{j}$ is incoming and $g_{j}$ is outgoing, but w.r.t. $\mathrm{C}_{j-1}, f_{j}$ is outgoing and $g_{j}$ is incoming.

The boundary conditions on the top and bottom and interior (if existing) boundaries could be either Neumann or Dirichlet, or any combination, but they need to be consistent with the geometry periodicity. Moreover, these boundary conditions should guarantee the well-posedness of the Helmholtz Equation (2.1) on the union of any finite number of periodic cells, if the incoming Sommerfeld data are prescribed on its left and right boundaries. We remark that these constraints are in fact very mild thanks to the Holmgren uniqueness Thm. [7, Sec. 5.3]. In this paper, if not specified explicitly, we assume homogeneous Neumann boundary conditions at the top and bottom boundaries.

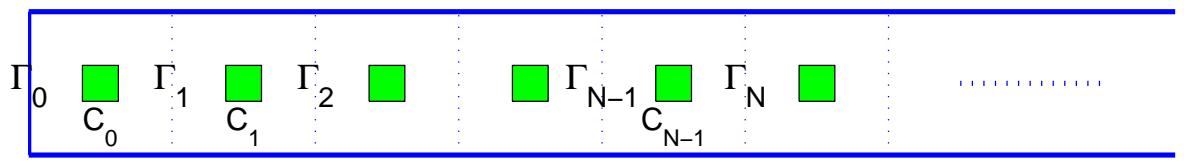

FIG. 2.1. Schematic view of a semi-infinite periodic array. $\mathrm{C}_{j}$ denotes the $j$-th periodic cell. $\Gamma_{j}$ is the left cell boundary of $\mathrm{C}_{j}$ and the right cell boundary of $\mathrm{C}_{j-1}$ (for $j \geq 1$ ).

To validate our methods which will be presented later on, three different periodic arrays (PA) will be considered, and we will refer to them as PA-One, PA-Two and 
PA-Three. All of them are composed of periodic cells with size of $1 \times 1$. More details are listed in the following:

- PA-One. Homogeneous waveguide. $n=1$.

- PA-Two. A hole of size $0.5 \times 0.5$ is made in the cell center. A homogeneous Dirichlet boundary condition is applied at the hole boundary. $n=1$.

- PA-Three. Inhomogeneous waveguide. $n=1+0.5 \cos (2 \pi x) \sin (2 \pi y)$.

To explore wave properties in a periodic array, it is usually helpful to look at dispersion diagram of the characteristic equation $-\Delta u=E n^{2} u$, restricted to a single periodic cell, say $\mathrm{C}_{0}$. The boundary conditions at the left and right boundaries are pseudoperiodic, namely,

$$
\left.u\right|_{\Gamma_{1}}=\left.e^{i \theta} u\right|_{\Gamma_{0}},\left.\quad u_{x}\right|_{\Gamma_{1}}=\left.e^{i \theta} u_{x}\right|_{\Gamma_{0}},
$$

where $\theta$ belongs to $[0,2 \pi)$. For each $\theta$, there exists a sequence of real eigenvalues $E$, usually called energies. All energies w.r.t. $\theta$ then compose the dispersion diagram. The dispersion relation for PA-One, the homogeneous waveguide, can be obtained analytically as

$$
E_{j m}=j^{2} \pi^{2}+(\theta+2 \pi m)^{2} .
$$

This multi-valued function is plotted in figure 2.2. For PA-Two and PA-Three, no analytical expressions of dispersion relation are available, and a spatial discretization method has to be employed. We use the eighth-order quadrilateral FEM method with mesh sizes $\Delta x=\Delta y=0.125$ for all the numerical tests reported in this paper. The dispersion diagrams for PA-Two and PA-Three are shown in figures 2.3-2.4. An obvious observation could be made that unlike the homogeneous waveguide, there are some bands of energy values in the dispersion diagrams of PA-Two and PAThree that could not be reached for any $\theta$. Physically, waves with energy (here $k^{2}$ ) in these bands could not propagate in the medium. In this context, they are usually referred to as stop bands in the engineering literature. In fact it is exactly this remarkable property which makes periodic structures very useful, for example, they could be elaborately designed to act as frequency selecting modules in the microwave and optical engineering.

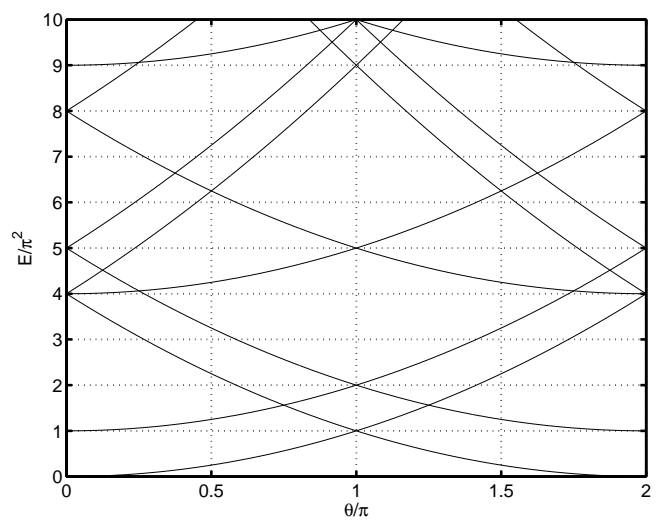

FIG. 2.2. Dispersion diagram of PA-One, a homogeneous waveguide. 


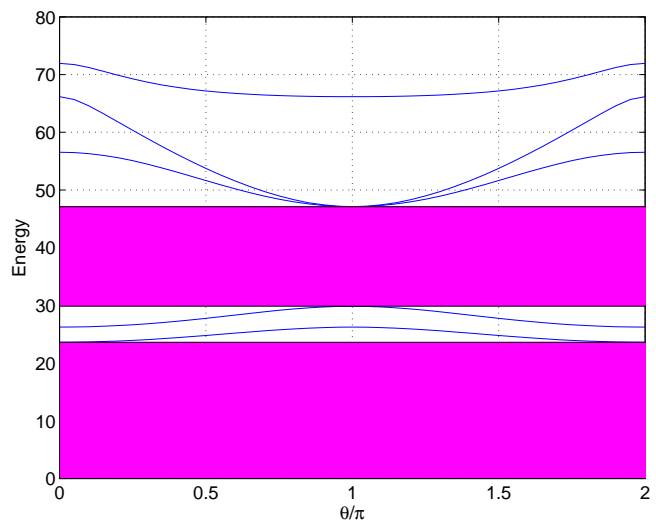

Fig. 2.3. Dispersion diagram of PA-Two. The first two stop bands are $\left(0,23.61_{ \pm 0.01}\right)$ and $\left(29.85_{ \pm 0.01}, 47.10_{ \pm 0.01}\right)$.

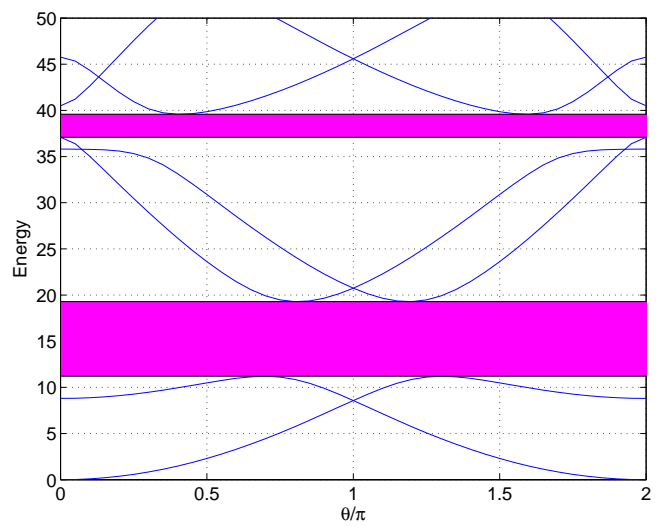

Fig. 2.4. Dispersion diagram of PA-Three. The first stop band is $\left(11.20_{ \pm 0.01}, 19.29_{ \pm 0.01}\right)$.

This paper is aimed at developing efficient numerical methods of evaluating exact scattering operators for semi-infinite periodic arrays at any real wavenumber $k$.

\section{The limiting absorption principle}

The first problem we are facing is to guarantee the well-posedness of the Helmholtz Equation (2.1), which naturally arises due to the absence of the radiation condition at infinity. Though well solved in the constant coefficient case for separable geometries [14], this problem largely remains open for the variable coefficient Helmholtz equation.

There are at least three methods of possibly deriving a unique solution of the Helmholtz equation in unbounded domains: asymptotic radiation condition, limiting absorption principle and limiting amplitude principle [22]. In this paper we resort to the limiting absorption principle (LABP). To understand this principle, let us first consider the following damped Helmholtz equation

$$
\Delta u^{\epsilon}+\left(k^{2}+i \epsilon\right) n^{2} u^{\epsilon}=0
$$


with the boundary condition

$$
\left.f_{0}\left(u^{\epsilon}\right) \equiv\left(\partial_{x}+i k\right) u^{\epsilon}\right|_{\Gamma_{0}}=f_{0}(u),
$$

where $\epsilon>0$ and $f_{0}(u) \in L^{2}\left(\Gamma_{0}\right)$ (take $f_{0}(u)$ as a unity). By the classical variational arguments, there exists a unique solution $u^{\epsilon} \in H^{1}(\Omega)$ and a bounded linear scattering operator $\mathcal{A}_{\text {inf }}^{\epsilon}$, which maps the Sommerfeld data $f_{0}\left(u^{\epsilon}\right)$ to another Sommerfeld data $g_{0}\left(u^{\epsilon}\right)$, namely,

$$
g_{0}\left(u^{\epsilon}\right)=\mathcal{A}_{\text {inf }}^{\epsilon} f_{0}\left(u^{\epsilon}\right) .
$$

The LABP is said to hold at $k>0$ if and only if for any $f_{0}(u) \in L^{2}\left(\Gamma_{0}\right)$, the solution $u^{\epsilon} \in H^{1}(\Omega)$ of the damped Helmholtz Equation (3.1) converges to a unique solution $u \in H_{l o c}^{1}(\Omega)$ of the Helmholtz Equation (2.1) as $\epsilon$ goes to zero, and the outgoing Sommerfeld data $g_{0}\left(u^{\epsilon}\right)$ also converge to the unique function $g_{0}(u)$. The function $u$ is then regarded as an $L A B P$ solution. The scattering operator of (2.1), denoted by $\mathcal{A}_{\text {inf }}$, is then defined as the limit of $\mathcal{A}_{\text {inf }}^{\epsilon}$, and it maps the Sommerfeld data $f_{0}(u)$ to the Sommerfeld data $g_{0}(u)$, i.e.,

$$
g_{0}(u)=\mathcal{A}_{\text {inf }} f_{0}(u)
$$

3.1. Homogeneous waveguide problem. Let us consider PA-One to obtain some insights on whether the LABP holds for our considered problem. In this case the separation of variables is available. Put

$$
u^{\epsilon}=\sum_{n=0}^{+\infty} u^{\epsilon, n} \cos (n \pi y), f_{0}(u)=\sum_{n=0}^{+\infty} f_{0}\left(u^{n}\right) \cos (n \pi y), g_{0}\left(u^{\epsilon}\right)=\sum_{n=0}^{+\infty} g_{0}\left(u^{\epsilon, n}\right) \cos (n \pi y),
$$

then (3.1) is transformed into a sequence of ODE problems:

$$
u_{x x}^{\epsilon, n}+\left(k^{2}+i \epsilon-n^{2} \pi^{2}\right) u_{x x}^{\epsilon, n}=0, f_{0}\left(u^{\epsilon, n}\right)=f_{0}\left(u^{n}\right), \forall n=0,1, \ldots
$$

The bounded solutions are

$$
u^{\epsilon, n}=\frac{f_{0}\left(u^{n}\right)}{i \sqrt{k^{2}+i \epsilon-n^{2} \pi^{2}}+i k} e^{i \sqrt{k^{2}+i \epsilon-n^{2} \pi^{2} x}} .
$$

Thus

$$
g_{0}\left(u^{\epsilon, n}\right)=\frac{i \sqrt{k^{2}+i \epsilon-n^{2} \pi^{2}}-i k}{i \sqrt{k^{2}+i \epsilon-n^{2} \pi^{2}}+i k} f_{0}\left(u^{n}\right)
$$

and

$$
g_{0}\left(u^{n}\right) \stackrel{\text { def }}{=} \lim _{\epsilon \rightarrow 0} g_{0}\left(u^{\epsilon, n}\right)=\frac{i \sqrt{k^{2}-n^{2} \pi^{2}}-i k}{i \sqrt{k^{2}-n^{2} \pi^{2}}+i k} f_{0}\left(u^{n}\right) .
$$

Besides, it is straightforward to verify that

$$
g_{0}\left(u^{\epsilon, n}\right)=g_{0}\left(u^{n}\right)+\left\{\begin{array}{cr}
\frac{2 \sqrt{i \epsilon} f_{0}\left(u^{n}\right)}{k}+O(\epsilon), & k=n \pi, \\
\frac{i k \epsilon f_{0}\left(u^{n}\right)}{\left(\sqrt{k^{2}-n^{2} \pi^{2}}+k\right)^{2} \sqrt{k^{2}-n^{2} \pi^{2}}}+O\left(\epsilon^{2}\right), & k \neq n \pi .
\end{array}\right.
$$


The expression (3.3) states that the convergence rate of $g_{0}\left(u^{\epsilon}\right)$ to

$$
g_{0}(u)=\sum_{n=0}^{+\infty} g_{0}\left(u^{n}\right) \cos (n \pi y)
$$

is of first order w.r.t. $\epsilon$ if $k$ is not equal to any $n \pi$ with $n \geq 0$, and if $k$ happens to equal some $n_{0} \pi$, which implies the resonance of the $n_{0}$-th mode in the $y$-direction, the convergence rate would degenerate to half order. But whatever the case is, the LABP holds at any $k>0$. Here, resonance means the existence of stationary wave solution which does not transport energy in the $x$-direction. In the dispersion diagrams, the square of resonance wavenumbers corresponds to those energies whose associated local dispersion curve has a zero slope. Obviously, $k$ is resonant if $k^{2}$ is an endpoint of any stop band. For example, $k=\sqrt{23.61_{ \pm 0.01}}, \sqrt{47.10_{ \pm 0.01}}$ is resonant for PA-Two, and $k=\sqrt{11.20_{ \pm 0.01}}, \sqrt{19.29_{ \pm 0.01}}$ for PA-Three.

Based on the above analysis, we conjecture that, under some mild conditions on the geometry and the refractive index function, the LABP holds at every $k>0$ for the general semi-infinite periodic arrays. Though we could not prove the conjecture in a rigorous mathematical way, its validity is supported by the numerical evidences which will be reported at the end of this section.

3.2. Scattering operator for the damped Helmholtz equation. The LABP itself suggests a method of deriving the exact StS scattering operator on the left boundary $\Gamma_{0}$ : first compute the scattering operator $\mathcal{A}_{\text {inf }}^{\epsilon}$ of the problem (3.1) for a given $\epsilon$, and then send $\epsilon$ to zero. In this subsection, we consider how to efficiently evaluate the scattering operator for the damped Helmholtz equation.

In [3] the authors proposed a fast evaluation method for the scattering operator of the damped Helmholtz Equation (3.1). The basic idea is as follows. For any $N>0$, the damped Helmholtz Equation (3.1) is well-posed on the domain $\cup_{j=0}^{N-1} \mathrm{C}_{j}$, with the incoming Sommerfeld data $f_{0}^{\epsilon}$ and $g_{N}^{\epsilon}$ prescribed at the boundaries $\Gamma_{0}$ and $\Gamma_{N}$. Thus there are four linear scattering operators $\mathcal{A}_{N}^{\epsilon}, \mathcal{B}_{N}^{\epsilon}, \mathcal{C}_{N}^{\epsilon}$ and $\mathcal{D}_{N}^{\epsilon}$ satisfying the so-called scattering relations

$$
g_{0}^{\epsilon}=\mathcal{A}_{N}^{\epsilon} f_{0}^{\epsilon}+\mathcal{B}_{N}^{\epsilon} g_{N}^{\epsilon}, f_{N}^{\epsilon}=\mathcal{C}_{N}^{\epsilon} f_{0}^{\epsilon}+\mathcal{D}_{N}^{\epsilon} g_{N}^{\epsilon} .
$$

Since $g_{N}^{\epsilon}$ goes to zero exponentially fast as $N$ goes to infinity, it is reasonable to expect that $\mathcal{A}_{N}^{\epsilon}$ converges and the limit is just the exact StS scattering operator $\mathcal{A}_{\text {inf }}^{\epsilon}$. To make this paper self-contained, we explain in Appendix A the fast doubling technique for deriving these scattering operators. This scheme needs only $O\left(\log _{2} N\right)$ operations.

Take PA-Two as an example. In Fig.3.1 we plot the relative errors of the scattering operators $\mathcal{A}_{N}^{\epsilon}$ compared to the reference operator $\mathcal{A}_{\text {ref }}^{\epsilon}$, which is obtained by using the doubling technique 20 times, i.e., $\mathcal{A}_{r e f}^{\epsilon}=\mathcal{A}_{N_{0}}^{\epsilon}$ with $N_{0}=2^{20}$. Since the eighth-order quadrilateral FEM is used with $\Delta x=\Delta y=0.125$, there are 65 unknowns getting involved at each cell boundary, and thus in the discrete level the scattering operators are approximated by matrices of rank $65 \times 65$. We could see that the doubling technique really leads to an efficient algorithm. Also notice that when $k^{2}$ lies in the stop bands, for example $k^{2}=23,31, \mathcal{A}_{N}$ itself converges as $N$ goes to infinity. This implies that when $k^{2}$ is in the stop bands, we could derive the StS scattering operator directly without considering the limiting procedure.

3.3. Extrapolation technique. Now we explain how to send $\epsilon$ to zero for the scattering operator $\mathcal{A}_{\text {inf }}^{\epsilon}$ to derive $\mathcal{A}_{\text {inf }}$. In terms of the expression (3.3), if $k$ is 


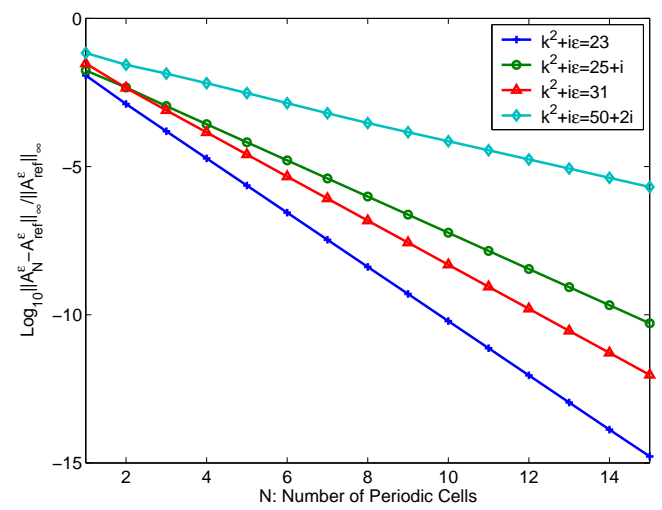

FIG. 3.1. Relative errors of $\mathcal{A}_{N}^{\epsilon}$ to the reference scattering operator $\mathcal{A}_{\text {ref }}^{\epsilon}$, which is equal to $\mathcal{A}_{N_{0}}^{\epsilon}$ with $N_{0}=2^{20}$. PA-Two.

not a resonance wavenumber, the exact StS scattering operator $\mathcal{A}_{\text {inf }}$ is expected to have an asymptotic expansion w.r.t. $\epsilon$ like

$$
\mathcal{A}_{\text {inf }}^{\epsilon}=\mathcal{A}_{\text {inf }}+\epsilon \mathcal{A}_{\text {inf }}^{(1)}+\epsilon^{2} \mathcal{A}_{\text {inf }}^{(2)}+\cdots .
$$

Thus in most cases, the convergence rate of the LABP is of first order. This is verified by the numerical evidences shown in figure 3.2. The convergence rate could be improved by the standard extrapolation technique, see [19] or Appendix B. In figure 3.3 we plot the errors of a reference scattering operator and the StS scattering operators computed by using extrapolation once. The reference solution is obtained by using extrapolation twice and setting a small damping parameter $\epsilon_{0}=0.00125$. One could see that the accuracy is greatly improved, and second order convergence rate can be clearly observed. We should also notice that if $k$ is close to a resonance wavenumber, for example $k^{2}=23.61,47.1$, the asymptotic convergence rate could only manifest well for sufficiently small damping parameters.

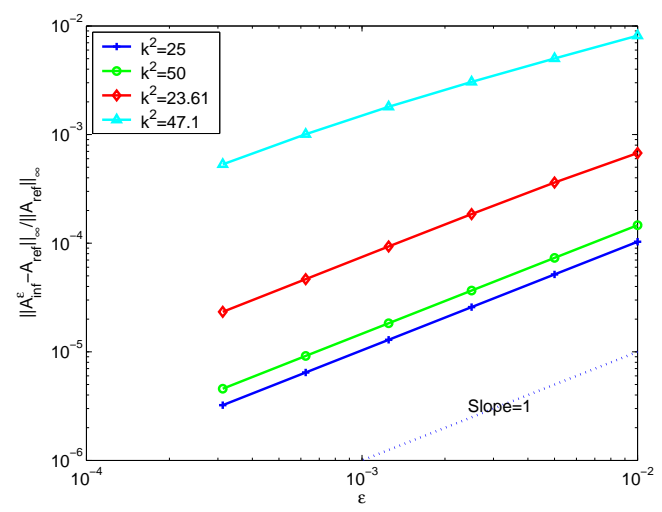

FIG. 3.2. Relative errors of the scattering operators $\mathcal{A}_{\text {inf }}^{\epsilon}$. The reference scattering operator $\mathcal{A}_{\text {ref }}$ is equal to $\mathcal{A}_{\text {inf }}^{\epsilon_{0}}$ with $\epsilon_{0}=10^{-7}$. PA-Two. 


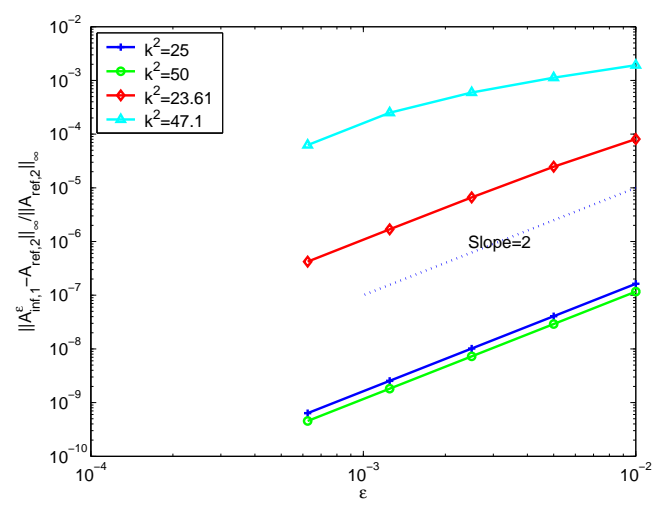

FIG. 3.3. Relative errors of the scattering operator $\mathcal{A}_{\text {inf }, 1}^{\epsilon}=-\mathcal{A}_{\text {inf }}^{\epsilon}+2 \mathcal{A}_{\text {inf }}^{\epsilon / 2}$. The reference operator $\mathcal{A}_{\text {ref }, 2}$ is obtained by using extrapolation technique twice with $\epsilon_{0}=0.00125$, i.e., $\mathcal{A}_{\text {ref }, 2}=$ $\mathcal{A}_{\text {inf }, 2}^{\epsilon_{0}}=\mathcal{A}_{\text {inf }}^{\epsilon_{0}} / 3-2 \mathcal{A}_{\text {inf }}^{\epsilon_{0} / 2}+8 \mathcal{A}_{\text {inf }}^{\epsilon_{0} / 4} / 3$. PA-Two.

\section{Asymptotic behavior of an LABP solution}

The last section shows that if $k$ is not a resonance wavenumber, the extrapolation technique could present very accurate scattering operators for the semi-infinite periodic arrays. This technique needs to evaluate the scattering operators for a sequence of $\epsilon$, which turns out to be costly. Besides, though the chance of $k$ being a resonance wavenumber is rare, if $k$ is close to a resonance wavenumber, the extrapolation method could not present very accurate result. Considering these two points, in this section we intend to develop a new method without resorting to the limiting procedure from the damped Helmholtz Equation (3.1) to the undamped one (2.1).

Recall in the last section that when $k^{2}$ lies in the stop bands, the exact scattering operator could be efficiently computed by the doubling technique without performing the limiting procedure. This is possible because the solution decays exponentially at infinity. When $k^{2}$ lies in the pass bands, in general an LABP solution cannot be expected to decay. Our basic idea is to separate those non-decaying waves and decaying waves and handle them by different means.

4.1. Floquet multipliers and Bloch wave solutions. First let us introduce some notions. Suppose $u$ and $v$ are two solutions of the Helmholtz Equation (2.1). Define the co-related energy flux of $u$ and $v$ as

$$
\mathcal{E}(u, v)=-2 i k\left[\left(u_{x}, v\right)_{\Gamma_{j}}-\left(u, v_{x}\right)_{\Gamma_{j}}\right]=(f(u), f(v))_{\Gamma_{j}}-(g(u), g(v))_{\Gamma_{j}}
$$

The energy flux of $u$ is defined as $\mathcal{E}(u, u)$, which is also equal to

$$
\mathcal{E}(u, u)=4 k \operatorname{Im} \int_{\Gamma_{j}} u_{x} \bar{u} d y .
$$

Note that the co-related energy flux does not rely on the choice of $\Gamma_{j}$, and $\mathcal{E}(\cdot, \cdot)$ defines a sesquilinear form.

A nontrivial solution $u$ of the Helmholtz Equation (2.1) is regarded as a Bloch wave solution associated with the Floquet multiplier $\alpha \in \mathbb{C}$, if $u$ satisfies two sequence of conditions

$$
\left.u\right|_{\Gamma_{i+1}}=\left.\alpha u\right|_{\Gamma_{i}},\left.\quad u_{x}\right|_{\Gamma_{i+1}}=\left.\alpha u_{x}\right|_{\Gamma_{i}}, \forall i=0,1, \cdots
$$


We denote by $\mathbb{F}$ the set of all Floquet multipliers. A Bloch wave solution is regarded as evanescent, traveling, or anti-evanescent if the associated Floquet multiplier $\alpha$ satisfies $|\alpha|<1,|\alpha|=1$, or $|\alpha|>1$. If $|\alpha|=1$, we regard $\alpha$ as a unitary Floquet multiplier. The set of all unitary Floquet multipliers is denoted by UTF. Note that the Floquet multiplier cannot be zero due to the Holmgren uniqueness theorem. For any $\alpha \in \mathbb{F}$, all associated Bloch wave solutions together with zero function form a linear space $\mathbb{E}_{\alpha}$, which will be referred to as an $\alpha$-eigenspace. Here we list some properties of Floquet multiplies and associated Bloch wave solutions (see [10, 11]):

1. If $\alpha \in \mathbb{F}$, then $1 / \alpha \in \mathbb{F}$ as well.

2. $\mathbb{U F}$ is a finite set. For any $\alpha \in \mathbb{U F}, N_{\alpha}=\operatorname{dim} \mathbb{E}_{\alpha}<+\infty$.

3. Given two Floquet multipliers $\alpha_{j}$ and $\alpha_{k}$, and two Bloch wave solutions $\varphi_{j} \in$ $\mathbb{E}_{\alpha_{j}}$ and $\varphi_{k} \in \mathbb{E}_{\alpha_{k}}$, if $\alpha_{j} \alpha_{k}^{*} \neq 1$, then $\mathcal{E}\left(\varphi_{j}, \varphi_{k}\right)=0$. Here the superscript $(\cdot)^{*}$ stands for the conjugate operator.

4. If $u$ is an LABP solution, then $u$ satisfies the energy criteria, say, the energy flux of $u$ is nonnegative.

There are two different methods for computing the Floquet multipliers and associated Bloch wave solutions. The first method utilizes the scattering relations (3.4) for $N=1$ and $\epsilon=0$, namely,

$$
g_{0}=\mathcal{A}_{1} f_{0}+\mathcal{B}_{1} g_{1}, f_{1}=\mathcal{C}_{1} f_{0}+\mathcal{D}_{1} g_{1} .
$$

In terms of (4.1) we have

$$
g_{1}=\alpha g_{0}, f_{1}=\alpha f_{0} .
$$

Substituting the above expressions into (4.2) gives

$$
\left(\begin{array}{ll}
-\mathcal{A}_{1} & I \\
-\mathcal{C}_{1} & 0
\end{array}\right)\left(\begin{array}{l}
f_{0} \\
g_{0}
\end{array}\right)=\alpha\left(\begin{array}{cc}
0 & \mathcal{B}_{1} \\
-I & \mathcal{D}_{1}
\end{array}\right)\left(\begin{array}{l}
f_{0} \\
g_{0}
\end{array}\right)
$$

This means that the Floquet multiplier $\alpha$ is a generalized eigenvalue of the above eigenvalue problem. The associated generalized eigenvector $\left(f_{0}, g_{0}\right)^{\top}$ is composed of two Sommerfeld data related to some $\alpha$-Bloch wave solutions $\varphi$. On $\mathrm{C}_{0}, \varphi$ satisfies the Helmholtz Equation (2.1) together with boundary conditions

$$
f_{0}(u)=f_{0}, g_{1}(u)=\alpha g_{0} .
$$

The second method resorts to a quadratic eigenvalue problem. Put

$$
\alpha=e^{\sigma L}, u=e^{\sigma x} \varphi, \operatorname{Im} \sigma \in\left[-\frac{\pi}{L}, \frac{\pi}{L}\right),
$$

where $L$ is the period, then $\alpha$ is a Floquet multiplier of the Helmholtz Equation (2.1) if and only if $\sigma$ solves the quadratic eigenvalue problem

$$
\left(\Delta+k^{2} n^{2}\right) \varphi+2 \sigma \partial_{x} \varphi+\sigma^{2} \varphi=0,
$$

where $\varphi$ is an eigenfunction which is $L$-periodic in the $x$-direction.

The most remarkable advantage of the first method lies in the fact that it involves much fewer degrees of freedom in the discrete level, since the Sommerfeld data is only defined on the cell boundary. This is indeed the main reason we adopt the first method in the following numerical tests. But the second method has its own merits. The eigenvalues of the quadratic eigenvalue problem come in pairs $(\sigma,-\sigma)$. This property can be maintained in the discrete level by a suitable projection method [21]. Comparatively, it is not obvious how to achieve this for the first method. This issue is now under investigation. 
4.2. Asymptotic behavior of an LABP solution. An LABP solution $u$ cannot include the anti-evanescent Bloch waves, thus asymptotically $u$ contains only traveling Bloch waves. It is known that not every traveling Bloch wave is an LABP solution. We need to pick out those traveling Bloch waves which are compatible with the LABP. It is already known that any LABP solution of (2.1) must satisfy the energy criteria, but this criteria is not sufficient to identify an LABP solution. To clarify this point, let us consider the homogeneous waveguide problem.

Suppose $k=\pi$. Then the traveling Bloch wave space is

$$
\operatorname{Span}\left\{e^{-i \pi x}, e^{i \pi x}, \cos (\pi y)\right\} .
$$

If the $x$-period $L$ is set as a non-integer positive number, then we get three unitary Floquet multipliers: $e^{-i \pi L}$ associated with $\operatorname{Span}\left\{e^{-i \pi x}\right\}, e^{i \pi L}$ with $\operatorname{Span}\left\{e^{i \pi x}\right\}$ and, 1 with $\operatorname{Span}\{\cos (\pi y)\}$. Since

$$
\begin{aligned}
& \mathcal{E}\left(e^{-i \pi x}, e^{-i \pi x}\right)=\left.4 \pi \operatorname{Im} \int_{0}^{1}\left(-i \pi e^{-i \pi x}\right) e^{i \pi x} d y\right|_{x=0}=-4 \pi^{2}, \\
& \mathcal{E}\left(e^{i \pi x}, e^{i \pi x}\right)=\left.4 \pi \operatorname{Im} \int_{0}^{1}\left(i \pi e^{i \pi x}\right) e^{-i \pi x} d y\right|_{x=0}=4 \pi^{2}, \\
& \mathcal{E}(\cos (\pi y), \cos (\pi y))=\left.4 \pi \operatorname{Im} \int_{0}^{1}(0) e^{i \pi x} d y\right|_{x=0}=0,
\end{aligned}
$$

we come to the correct conclusion that according to the energy criteria, $e^{i \pi x}$ and $\cos (\pi y)$ are LABP solutions, while $e^{-i \pi x}$ is not.

The above reasoning ceases to work if we take the period $L$ as an integer. For example, take $L=1$. In this case there are two unitary Floquet multipliers 1 and -1 , namely,

$$
\begin{aligned}
& \alpha_{1}=-1 \longleftrightarrow \mathbb{E}_{\alpha_{1}}=\operatorname{Span}\left\{e^{-i \pi x}, e^{i \pi x}\right\}, \\
& \alpha_{2}=1 \longleftrightarrow \mathbb{E}_{\alpha_{2}}=\operatorname{Span}\{\cos (\pi y)\} .
\end{aligned}
$$

$\mathbb{E}_{\alpha_{2}}$ represents a resonance wave space, and two-dimensional space $\mathbb{E}_{\alpha_{1}}$ contains both the left-going and right-going traveling Bloch waves. Then how to classify these two kind of waves? One might say that the energy criteria still works, since obviously the Bloch wave $e^{i \pi x}$ is right-going, and $e^{-i \pi x}$ is left-going. But the question lies in the fact that $\mathbb{E}_{\alpha_{1}}$ may have a different basis representation, for example,

$$
\mathbb{E}_{\alpha_{1}}=\operatorname{Span}\left\{e^{-i \pi x}+2 e^{i \pi x}, e^{-i \pi x}+3 e^{i \pi x}\right\}=\operatorname{Span}\left\{e^{i \pi x}+2 e^{-i \pi x}, e^{i \pi x}+3 e^{-i \pi x}\right\} .
$$

For the first representation, both basis wave functions have positive energy fluxes, and for the second, both have negative energy fluxes. Using the energy criteria naively would yield contradictory conclusions. The lesson thus given to us is that if the eigenspace is multi-dimensional, generally we could not identify an LABP traveling wave solution by merely checking its energy flux.

The above problem becomes more severe if we take $L=2$. In this case there is only one unitary Floquet multiplier

$$
\alpha=1 \longleftrightarrow \mathbb{E}_{\alpha}=\operatorname{Span}\left\{e^{-i \pi x}, e^{i \pi x}, \cos (\pi y)\right\} .
$$

It is not difficult to find different basis representations for $\mathbb{E}_{\alpha}$, which have completely different signs of energy flux. On a conclusion, if $\alpha$ is a unitary Floquet multiplier 
and its associated eigenspace $\mathbb{E}_{\alpha}$ is multi-dimensional, we have to resort to a more complete criteria to identify LABP Bloch wave solutions. We should remark here that for a three-dimensional waveguide problem, the chance to have multi-dimensional $\mathbb{E}_{\alpha}$ is absolutely not rare, though it seems true for two-dimensional waveguide problems.

Suppose $\alpha \in \mathbb{U F}$, and $\left\{\varphi_{j}\right\}_{j=1}^{N_{\alpha}}$ constitute a set of basis functions of $\mathbb{E}_{\alpha}$, orthonormal w.r.t. the $n^{2}$-weighted inner product $(\cdot, \cdot)_{n^{2}}$ defined as

$$
\left(\varphi_{j}, \varphi_{k}\right)_{n^{2}}=\int_{\mathrm{C}_{0}} n^{2} \varphi_{j} \bar{\varphi}_{k} d y .
$$

We define the $N_{\alpha} \times N_{\alpha}$ energy flux matrix $M=\left(m_{j k}\right)$ as

$$
m_{j k}=\mathcal{E}\left(\varphi_{j}, \varphi_{k}\right), \forall j, k=1,2, \cdots, N_{\alpha} .
$$

It is easy to verify that $M$ is a Hermitian matrix, which implies the existence of a unitary matrix $U$, such that

$$
U^{\top} M \bar{U}=\Lambda=\operatorname{diag}\left(\lambda_{1}, \lambda_{2}, \ldots, \lambda_{N_{\alpha}}\right),
$$

where $\lambda_{j}$ are real eigenvalues of $M$ ordered by

$$
\lambda_{1} \geq \lambda_{2} \geq \cdots \geq \lambda_{m_{1}}>0=\lambda_{m_{1}+1}=\cdots=\lambda_{m_{2}}=0>\lambda_{m_{2}+1} \geq \cdots \geq \lambda_{N_{\alpha}} .
$$

Then we could introduce a new set of basis functions $\left\{\psi_{j}\right\}_{j=1}^{N_{\alpha}}$ as

$$
\left(\psi_{1}, \ldots, \psi_{N_{\alpha}}\right)=\left(\varphi_{1}, \ldots, \varphi_{N_{\alpha}}\right) U
$$

which will be referred to as a canonical set of basis functions of $\mathbb{E}_{\alpha}$. The eigenfunction space $\mathbb{E}_{\alpha}$ is then separated into three parts, i.e.,

$$
\mathbb{E}_{\alpha}=\mathbb{R}_{\alpha} \oplus \mathbb{S}_{\alpha} \oplus \mathbb{L}_{\alpha}
$$

with

$$
\mathbb{R}_{\alpha}=\operatorname{Span}\left\{\psi_{1}, \cdots, \psi_{m_{1}}\right\}, \mathbb{S}_{\alpha}=\operatorname{Span}\left\{\psi_{m_{1}+1}, \cdots, \psi_{m_{2}}\right\}, \mathbb{L}_{\alpha}=\operatorname{Span}\left\{\psi_{m_{2}+1}, \cdots, \psi_{N_{\alpha}}\right\} .
$$

Proposition 4.1. For any $\alpha \in \mathbb{E}_{\alpha},\left\{\lambda_{j}\right\}_{j=1}^{N_{\alpha}}$ are invariant quantities, and $\mathbb{R}_{\alpha}, \mathbb{S}_{\alpha}$ and $\mathbb{L}_{\alpha}$ are invariant subspaces of $\mathbb{E}_{\alpha}$. Besides, for any $\varphi_{1} \in \mathbb{R}_{\alpha}, \varphi_{2} \in \mathbb{S}_{\alpha}, \varphi_{3} \in \mathbb{L}_{\alpha}$, we have

$$
\mathcal{E}\left(\varphi_{1}, \varphi_{1}\right)>0, \mathcal{E}\left(\varphi_{2}, \varphi_{2}\right)=0, \mathcal{E}\left(\varphi_{3}, \varphi_{3}\right)<0, \mathcal{E}\left(\varphi_{m}, \varphi_{n}\right)=0, \forall m \neq n
$$

For the homogeneous waveguide problem, it is straightforward to verify that $\mathbb{R}_{\alpha}$ is the admissible LABP Bloch wave space with positive energy flux. $\mathbb{S}_{\alpha}$ is the resonance wave space, which is also compatible with the LABP. Note that if $\mathbb{S}_{\alpha}$ is excluded from the LABP solution space, the Helmholtz Equation (2.1) would lose solvability for some Sommerfeld data $f_{0}$, thus well-posedness is destroyed. Based on these facts, for a general semi-infinite periodic array, we make the following conjecture.

Conjecture 4.2. Suppose $\alpha_{1}, \cdots, \alpha_{M}$ are all unitary Floquet multipliers, and $\left\{\varphi_{1}^{\alpha_{j}}, \cdots, \varphi_{M_{\alpha_{j}}}^{\alpha_{j}}\right\}$ constitute a set of basis functions of $\mathbb{R}_{\alpha_{j}} \oplus \mathbb{S}_{\alpha_{j}}$. Then asymptotically, any LABP solution $u$ lies in the space

$$
\operatorname{Span}\left\{\varphi_{k}^{\alpha_{j}} \mid j=1, \cdots, M, k=1, \cdots, M_{\alpha_{j}}\right\} .
$$

The above conjecture thus gives a complete criteria for identifying an LABP solution. Though we are unable to prove it at this time, its validity will be shown by the numerical tests given in Sec. 5 . 
4.3. Asymptotic method of evaluating the scattering operators. Based on Conjecture 4.2, we know when $N$ is large, asymptotically,

$$
f_{N}(u) \approx \sum_{j=1}^{M} \sum_{k=1}^{M_{\alpha_{j}}} t_{k}^{j} f_{0}\left(\varphi_{k}^{\alpha_{j}}\right), g_{N}(u) \approx \sum_{j=1}^{M} \sum_{k=1}^{M_{\alpha_{j}}} t_{k}^{j} g_{0}\left(\varphi_{k}^{\alpha_{j}}\right) .
$$

Or in an abbreviated vector form,

$$
f_{N}(u) \approx F T, g_{N}(u) \approx G T,
$$

where

$$
F=\left(F_{1}, \cdots, F_{M}\right), G=\left(G_{1}, \cdots, G_{M}\right), T=\left(T_{1}, \cdots, T_{M}\right)^{\top}
$$

with

$$
F_{j}=\left(f_{0}\left(\varphi_{1}^{\alpha_{j}}\right), \cdots, f_{0}\left(\varphi_{M_{\alpha_{j}}}^{\alpha_{j}}\right)\right), G_{j}=\left(g_{0}\left(\varphi_{1}^{\alpha_{j}}\right), \cdots, g_{0}\left(\varphi_{M_{\alpha_{j}}}^{\alpha_{j}}\right)\right), T_{j}=\left(t_{1}^{\alpha_{j}}, \cdots, t_{M_{\alpha_{j}}}^{\alpha_{j}}\right) .
$$

Recall the scattering relations

$$
g_{0}(u)=\mathcal{A}_{N} f_{0}(u)+\mathcal{B}_{N} g_{N}(u), \quad f_{N}(u)=\mathcal{C}_{N} f_{0}(u)+\mathcal{D}_{N} g_{N}(u) .
$$

Using (4.5) $T$ could be derived by the least square method as

$$
T \approx\left(F-\mathcal{D}_{N} G\right)^{-1} \mathcal{C}_{N} f_{0}(u) .
$$

Here, $(\cdot)^{-1}$ denotes the pseudo-inverse operator. We then have

$$
g_{0}(u)=\mathcal{A}_{N} f_{0}(u)+\mathcal{B}_{N} g_{N}(u) \approx\left(\mathcal{A}_{N}+\mathcal{B}_{N} G\left(F-\mathcal{D}_{N} G\right)^{-1} \mathcal{C}_{N}\right) f_{0}(u),
$$

which means that by putting

$$
\tilde{\mathcal{A}}_{N}=\mathcal{A}_{N}+\mathcal{B}_{N} G\left(F-\mathcal{D}_{N} G\right)^{-1} \mathcal{C}_{N},
$$

the limit of $\tilde{\mathcal{A}}_{N}$ would give the exact StS scattering operator $\mathcal{A}_{\text {inf }}$ on the left boundary $\Gamma_{0}$.

The key point to implement the above algorithm is to derive a canonical set of basis functions for all unitary Floquet multipliers. More explicitly, we need to compute the functions $F_{j}$ and $G_{j}$ defined in (4.6) whose columns span the Sommerfeld datum space of all LABP solution of $\mathbb{E}_{\alpha}$. This can be achieved within several steps:

1. Solve the generalized eigenvalue problem (4.3) to obtain all (may be same) unitary Floquet multipliers $\left\{\alpha_{j}\right\}_{j=1}^{M}$ and their associated generalized eigenvector $\left(f_{0}^{j}, g_{0}^{j}\right)^{\top}$.

2. If $\alpha_{j}$ is simple, i.e., $N_{\alpha_{j}}=1$, compute the energy flux of the Bloch wave solution $\varphi^{j}$ associated with the Sommerfeld data $\left(f_{0}^{j}, g_{0}^{j}\right)$ by

$$
\mathcal{E}\left(\varphi^{j}, \varphi^{j}\right)=\left(f_{0}^{j}, f_{0}^{j}\right)_{\Gamma_{0}}-\left(g_{0}^{j}, g_{0}^{j}\right)_{\Gamma_{0}} .
$$

If $\mathcal{E}\left(\varphi^{j}, \varphi^{j}\right) \geq 0$, then $\varphi^{j}$ is an admissible LABP traveling Bloch wave. Thus $F_{j}=\left(f_{0}^{j}\right), G_{j}=\left(g_{0}^{j}\right)$. Otherwise, $F_{j}=G_{j}=\emptyset$. Note that in this case we do not need to compute $\varphi^{j}$ explicitly.

3. If $\alpha_{j}$ is multiple, i.e., $N_{\alpha_{j}}>1$, derive a set of basis functions $\left\{\varphi_{1}^{\alpha_{j}}, \cdots, \varphi_{M_{\alpha_{j}}}^{\alpha_{j}}\right\}$ of the admissible LABP Bloch wave space $\mathbb{R}_{\alpha_{j}} \oplus \mathbb{S}_{\alpha_{j}}$ with the method explained in subsection 4.2. Then set $F_{j}=\left(F_{j}^{1}, \cdots, F_{j}^{M_{\alpha_{j}}}\right)$ and $G_{j}=\left(G_{j}^{1}, \cdots, G_{j}^{M_{\alpha_{j}}}\right)$ with

$$
F_{j}^{k}=f_{0}\left(\varphi_{k}^{\alpha_{j}}\right), G_{j}^{k}=g_{0}\left(\varphi_{k}^{\alpha_{j}}\right), \forall k=1, \cdots, M_{\alpha_{j}} .
$$


4.4. Why not modal expansion? In the first step of the algorithm for computing the functions $F_{j}$ and $G_{j}$ defined in (4.6), we need to compute all unitary Floquet multipliers. So far an efficient algorithm for arriving at this is still under investigation. In the numerical tests of next section, we simply employ the traditional QZ-algorithm [13] to compute all Floquet multipliers and their associated eigenfunctions. Then a natural question is why not develop a modal expansion method like the following:

1. Compute all Floquet multipliers $\alpha_{j}$ and associated eigenvectors $\left(f_{0}^{j}, g_{0}^{j}\right)^{\top}$ by solving the generalized eigenvalue problem (4.3);

2. For any unitary Floquet multiplier $\alpha_{j}$, use the algorithm in the last subsection to derive $F_{j}$ and $G_{j}$;

3. For any Floquet multiplier $\alpha_{j}$ with $\left|\alpha_{j}\right|<1$, set $F_{j}=\left(f_{0}^{j}\right), G_{j}=\left(g_{0}^{j}\right)$;

4. Suppose $F=\left(\cdots, F_{j}, \cdots\right)$ spans the space $L^{2}\left(\Gamma_{0}\right)$. Set $G=\left(\cdots, G_{j}, \cdots\right)$. The scattering operator is then given as

$$
\mathcal{A}_{\text {inf }}=G F^{-1}: f_{0} \longrightarrow g_{0} .
$$

Despite many theoretical issues which are still mysterious to us, for example, the independence of $\left\{F_{j}\right\}$ and the completeness of $\left\{F_{j}\right\}$ in $L^{2}\left(\Gamma_{0}\right)$, two computationally relevant issues prevent us from considering the modal expansion method. First, in general $\left\{F_{j}\right\}$ are not orthogonal in any sense. Second, as suggested by our numerical tests, the computation of Sommerfeld data associated with Floquet multiplier of small modulus is inaccurate and ill-conditioned. These two factors imply that the evaluation of the inverse operator $F^{-1}$ thus $\mathcal{A}_{\text {inf }}$ would be very costly and badly ill-conditioned when the number of degrees of freedom on each cell boundary is large.

\section{Numerical tests}

In this section we will report some numerical tests. First we consider PA-One. In this case the analytical scattering operator is available. For the $n$-th mode in the $y$-direction, the exact StS mapping is given as in (3.2). The computed scattering operator, no matter which method is employed, is diagonalizable. In Table 5.1 we list the errors of scattering operators for different $y$-modes computed with the asymptotic method.

We see that generally the asymptotic method presents very accurate results, though a little bit worse but still satisfactory at the resonance wavenumber. For example, when $k=\pi$, the first $y$-mode $(n=1)$ is resonant. The error of computed scattering operator is of order $10^{-6}$, while others are at least of order $10^{-9}$. In Table 5.2 we list the errors of scattering operators computed by the extrapolation method. Two times of extrapolation have been performed with $\epsilon_{0}=0.00125$, namely, the computed scattering operator is $\mathcal{A}_{\text {inf }}^{\epsilon_{0}} / 3-2 \mathcal{A}_{\text {inf }}^{\epsilon_{0} / 2}+8 \mathcal{A}_{\text {inf }}^{\epsilon_{0} / 4} / 3$, where each $\mathcal{A}_{\text {inf }}^{\epsilon}$ is computed using the doubling technique sufficiently many times. We see that except at the resonance wavenumbers, the extrapolation method presents results nearly of the same quality as those derived by the asymptotic method. When resonance occurs, the asymptotic method is superior to the extrapolation method.

For periodic arrays PA-Two and PA-Three, no analytical expression is available on the exact scattering operators. We compare the scattering operators computed with two proposed methods, and list the relative errors in Table 5.3. Since the eighthorder FEM is used for the spatial discretization with mesh sizes $\Delta x=\Delta y=0.125$, in the discrete level there are 65 degrees of freedom at each cell boundary and the 
scattering operators are approximated by $65 \times 65$ matrices. The errors are evaluated with infinity norms. From Table 5.3 we could conclude that in principle these two methods bring the same results, which suggests that our asymptotic method is compatible with the limiting absorption principle. We could also observe that when $k$ is away from the resonance wavenumber, these two methods present results nearly of same quality. But their difference becomes large when $k$ turns close to the resonance wavenumber. Considering the results for the homogeneous waveguide problem, we believe that this happens since in this case the extrapolation method presents much worse results compared with the asymptotic method.

\begin{tabular}{c|c|c|c|c|c}
\hline & $n=0$ & $n=1$ & $n=2$ & $n=3$ & $n=4$ \\
\hline$k=\pi$ & $1.50 \times 10^{-9}$ & $7.58 \times 10^{-6}$ & $2.13 \times 10^{-12}$ & $5.44 \times 10^{-13}$ & $2.28 \times 10^{-13}$ \\
\hline$k=\frac{5 \pi}{4}$ & $4.60 \times 10^{-9}$ & $1.78 \times 10^{-9}$ & $3.52 \times 10^{-12}$ & $8.74 \times 10^{-13}$ & $3.24 \times 10^{-13}$ \\
\hline$k=\sqrt{2} \pi$ & $7.02 \times 10^{-12}$ & $1.07 \times 10^{-9}$ & $1.31 \times 10^{-11}$ & $2.80 \times 10^{-12}$ & $1.00 \times 10^{-12}$ \\
\hline$k=\sqrt{3} \pi$ & $5.91 \times 10^{-13}$ & $9.44 \times 10^{-13}$ & $3.23 \times 10^{-12}$ & $5.40 \times 10^{-13}$ & $2.10 \times 10^{-13}$ \\
\hline
\end{tabular}

TABLE 5.1. Errors of scattering operators for different $y$-modes of homogeneous waveguide. The scattering operators are computed by the asymptotic method.

\begin{tabular}{c|c|c|c|c|c}
\hline & $n=0$ & $n=1$ & $n=2$ & $n=3$ & $n=4$ \\
\hline$k=\pi$ & $5.03 \times 10^{-9}$ & $5.68 \times 10^{-3}$ & $2.26 \times 10^{-12}$ & $7.51 \times 10^{-13}$ & $2.39 \times 10^{-13}$ \\
\hline$k=\frac{5 \pi}{4}$ & $3.53 \times 10^{-12}$ & $7.30 \times 10^{-12}$ & $3.43 \times 10^{-12}$ & $8.22 \times 10^{-13}$ & $2.74 \times 10^{-13}$ \\
\hline$k=\sqrt{2} \pi$ & $6.91 \times 10^{-12}$ & $1.52 \times 10^{-8}$ & $1.26 \times 10^{-11}$ & $2.99 \times 10^{-12}$ & $1.06 \times 10^{-12}$ \\
\hline$k=\sqrt{3} \pi$ & $1.07 \times 10^{-12}$ & $1.49 \times 10^{-12}$ & $3.40 \times 10^{-12}$ & $5.82 \times 10^{-13}$ & $2.37 \times 10^{-13}$ \\
\hline
\end{tabular}

TABLE 5.2. Errors of scattering operators for different $y$-modes of homogeneous waveguide. The scattering operators are computed by extrapolating $\mathcal{A}_{\text {inf }}^{\epsilon}$ twice with $\epsilon_{0}=0.00125$, namely, the computed scattering operators are $\mathcal{A}_{\text {inf }}^{\epsilon_{0}} / 3-2 \mathcal{A}_{\text {inf }}^{\epsilon_{0} / 2}+8 \mathcal{A}_{\text {inf }}^{\epsilon_{0} / 4} / 3$.

\begin{tabular}{c|c|c|c|c}
\hline PA-Two & $k^{2}=25$ & $k^{2}=50$ & $k^{2}=23.61$ & $k^{2}=47.1$ \\
\hline Relative errors & $1.31 \times 10^{-12}$ & $3.26 \times 10^{-12}$ & $3.89 \times 10^{-8}$ & $6.76 \times 10^{-5}$ \\
\hline PA-Three & $k^{2}=5$ & $k^{2}=25$ & $k^{2}=11.20$ & $k^{2}=19.29$ \\
\hline Relative errors & $9.58 \times 10^{-13}$ & $9.26 \times 10^{-13}$ & $7.16 \times 10^{-9}$ & $6.23 \times 10^{-10}$ \\
\hline
\end{tabular}

TABLE 5.3. Relative errors between the scattering operators computed by the extrapolation method and the asymptotic method. The scattering operators by extrapolation method are $\mathcal{A}_{\text {inf }}^{\epsilon_{0}} / 3-$ $2 \mathcal{A}_{\text {inf }}^{\epsilon_{0} / 2}+8 \mathcal{A}_{\text {inf }}^{\epsilon_{0} / 4} / 3$ with $\epsilon_{0}=0.00125$.

Conclusion. In this paper we have considered scattering operators of Sommerfeld-to-Sommerfeld type for periodic Helmholtz equations in semi-infinite periodic arrays. Since no radiation condition could be specified explicitly at infinity, the Helmholtz equation is not complete. To solve this problem we employ the limiting absorption principle. We have proposed two different methods, extrapolation method and asymptotic method, to evaluate scattering operators. The extrapolation method is based on the doubling technique and the limiting procedure, while the asymptotic 
method is based on a conjecture for the asymptotic behavior of LABP solutions. The equivalence of these two methods has been supported by the numerical tests, though its rigorous proof is not available at this time. Numerical tests have also shown that when the wavenumber becomes resonant, the asymptotic method could present better results than the extrapolation method.

We expect the asymptotic method could be extended to more complicated wavelike equations, such as Maxwell's equations and elastic wave equations on periodic arrays. Besides, we have left the relevant theoretical problems largely open in this paper. These issues are now under investigation.

Appendix A. On the vertical boundary segments $\Gamma_{i}$ we define two Sommerfeld data as

$$
f_{i}=\left.\left(\partial_{x}+i k\right) u\right|_{\Gamma_{i}}, \quad g_{i}=\left.\left(\partial_{x}-i k\right) u\right|_{\Gamma_{i}} .
$$

For any given boundary data $f_{i}$ and $g_{i+k}, i \geq 0, k \geq 1$, the Helmholtz Equation (2.1) is uniquely solvable on $\cup_{l=i}^{i+k-1} C_{l}$, cf. [3, Lem. A]. Due to the assumption on the boundary conditions, there exists four linear operators $\mathcal{A}_{k}, \mathcal{B}_{k}, \mathcal{C}_{k}$ and $\mathcal{D}_{k}$ satisfying

$$
g_{i}=\mathcal{A}_{k} f_{i}+\mathcal{B}_{k} g_{i+k}, \quad f_{i+k}=\mathcal{C}_{k} f_{i}+\mathcal{D}_{k} g_{i+k} .
$$

From the numerical point of view, these operators can be derived by an appropriate spatial discretization in the domain $\cup_{k=0}^{j-1} C_{k}$. But if $k$ is large, a vast number of unknowns would get involved, which leads to a high computational effort. As revealed in [3], these operators can be obtained very efficiently with a smart doubling technique.

The essential idea of this doubling procedure is based on the following recursive relations. Suppose for $k \in\{m, n\}$, the operators $\mathcal{A}_{k}, \mathcal{B}_{k}, \mathcal{C}_{k}$ and $\mathcal{D}_{k}$ have already been obtained. By (A.2) we have

$$
\begin{aligned}
& g_{i}=\mathcal{A}_{m}\left(\mathcal{C}_{n} f_{i-n}+\mathcal{D}_{n} g_{i}\right)+\mathcal{B}_{m} g_{i+m}, \\
& f_{i}=\mathcal{C}_{n} f_{i-n}+\mathcal{D}_{n}\left(\mathcal{A}_{m} f_{i}+\mathcal{B}_{m} g_{i+m}\right) .
\end{aligned}
$$

It is easy to prove that $I-\mathcal{A}_{m} \mathcal{D}_{n}$ and $I-\mathcal{D}_{n} \mathcal{A}_{m}$ ( $I$ denotes the identity operator) are invertible, cf. [3, Lem. B]. Thus,

$$
g_{i}=\mathcal{A}_{n, m}^{*} f_{i-n}+\mathcal{B}_{n, m}^{*} g_{i+m}, \quad f_{i}=\mathcal{C}_{n, m}^{*} f_{i-n}+\mathcal{D}_{n, m}^{*} g_{i+m},
$$

where

$$
\begin{aligned}
& \mathcal{A}_{n, m}^{*}=\left(I-\mathcal{A}_{m} \mathcal{D}_{n}\right)^{-1} \mathcal{A}_{m} \mathcal{C}_{n}, \quad \mathcal{B}_{n, m}^{*}=\left(I-\mathcal{A}_{m} \mathcal{D}_{n}\right)^{-1} \mathcal{B}_{m} \\
& \mathcal{C}_{n, m}^{*}=\left(I-\mathcal{D}_{n} \mathcal{A}_{m}\right)^{-1} \mathcal{C}_{n}, \quad \mathcal{D}_{n, m}^{*}=\left(I-\mathcal{D}_{n} \mathcal{A}_{m}\right)^{-1} \mathcal{D}_{n} \mathcal{B}_{m}
\end{aligned}
$$

Substituting expressions (A.3) into (A.2) gives

$$
\begin{aligned}
& g_{i-n}=\mathcal{A}_{n} f_{i-n}+\mathcal{B}_{n}\left(\mathcal{A}_{n, m}^{*} f_{i-n}+\mathcal{B}_{n, m}^{*} g_{i+m}\right), \\
& f_{i+m}=\mathcal{C}_{m}\left(\mathcal{C}_{n, m}^{*} f_{i-n}+\mathcal{D}_{n, m}^{*} g_{i+m}\right)+\mathcal{D}_{m} g_{i+m},
\end{aligned}
$$

which imply that

$$
\begin{aligned}
& \mathcal{A}_{m+n}=\mathcal{A}_{n}+\mathcal{B}_{n} \mathcal{A}_{n, m}^{*}, \quad \mathcal{B}_{m+n}=\mathcal{B}_{n} \mathcal{B}_{n, m}^{*} \\
& \mathcal{C}_{m+n}=\mathcal{C}_{m} \mathcal{C}_{n, m}^{*}, \quad \mathcal{D}_{m+n}=\mathcal{D}_{m}+\mathcal{C}_{m} \mathcal{D}_{n, m}^{*}
\end{aligned}
$$

Hence, for any fixed cell number $N$, the operators $\mathcal{A}_{N}, \mathcal{B}_{N}, \mathcal{C}_{N}$, and $\mathcal{D}_{N}$ can be obtained by the following steps: 
1. Derive $\mathcal{A}_{1}, \mathcal{B}_{1}, \mathcal{C}_{1}$, and $\mathcal{D}_{1}$ by the cell analysis. If $N=1$, it is done;

2. Write $N$ into binary form $\left(j_{L} \cdots j_{0}\right)_{2}$, with $L=\left[\log _{2} N\right]$ and $j_{L}=1$;

3. Use the relations (A.4) $L$ times by setting $m=n=2^{k-1}$ to get $\mathcal{A}_{2^{k}}, \mathcal{B}_{2^{k}}, \mathcal{C}_{2^{k}}$, and $\mathcal{D}_{2^{k}}$ for $k=1, \ldots, L$;

4. For $l=L-1, \ldots, 0$, if $j_{l} \neq 0$, then use (A.4) by setting $m=\left(j_{L} \cdots j_{l+1} 0 \cdots 0\right)_{2}$ and $n=2^{l}$ to obtain $\mathcal{A}_{\left(j_{L} \cdots j_{l} 0 \cdots 0\right)_{2}}, \quad \mathcal{B}_{\left(j_{L} \cdots j_{l} \cdots \cdots\right)_{2}}, \quad \mathcal{C}_{\left(j_{L} \cdots j_{l} 0 \cdots 0\right)_{2}}$ and $\mathcal{D}_{\left(j_{L} \cdots j_{l} 0 \cdots 0\right)_{2}}$.

The above procedure uses (A.4) at most $2\left[\log _{2} N\right]$ times, and only $J$ times if $N=2^{J}$.

Appendix B. Suppose $T(\epsilon)$ converges to a required quantity $\tau_{0}$ as $\epsilon$ goes to zero, and for each fixed $\epsilon>0, T(\epsilon)$ can be computed with a prescribed algorithm, but CANNOT for $\epsilon=0$. Furthermore, suppose $T(\epsilon)$ admits an asymptotic expansion in powers of $\epsilon$ as

$$
T(\epsilon)=\tau_{0}+\tau_{1} \epsilon^{\gamma_{1}}+\tau_{2} \epsilon^{\gamma_{2}}+\cdots+\tau_{m} \epsilon^{\gamma_{m}}+\epsilon^{\gamma_{m+1}} \alpha_{m+1}(\epsilon),
$$

with

$$
0<\gamma_{1}<\gamma_{2}<\cdots<\gamma_{m+1} .
$$

Here the exponents $\gamma_{i}$ need not to be integers. The coefficients $\tau_{i}$ are independent of $\epsilon$, and the function $\alpha_{m+1}(\epsilon)$ is bounded as $\epsilon \rightarrow 0$.

As shown by the above expansion, $T(\epsilon)$ is only a $\gamma_{1}$-th order approximation of $\tau_{0}$. If we set

$$
T_{1}(\epsilon)=\frac{2^{\gamma_{1}} T(\epsilon / 2)-T(\epsilon)}{2^{\gamma_{1}}-1},
$$

then

$$
T_{1}(\epsilon)=\tau_{0}+\tau_{2}^{\prime} \epsilon^{\gamma_{2}}+\cdots+\tau_{m}^{\prime} \epsilon^{\gamma_{m}}+\epsilon^{\gamma_{m+1}} \alpha_{m+1}^{\prime}(\epsilon),
$$

where

$$
\tau_{i}^{\prime}=\frac{2^{\gamma_{1}-\gamma_{i}}-1}{2^{\gamma_{1}}-1} \tau_{i}, \forall i=2, \cdots, m, \quad \alpha_{m+1}^{\prime}(\epsilon)=\frac{2^{\gamma_{1}-\gamma_{m+1}} \alpha_{m+1}(\epsilon / 2)-\alpha_{m+1}(\epsilon)}{2^{\gamma_{1}}-1} .
$$

This means that $T_{1}(\epsilon)$ is a better approximation of $\tau_{0}$ with higher convergence rate $\gamma_{2}$. This simple technique of improving algorithm accuracy is called extrapolation, and it can be used successively for many times.

For the asymptotic expansion (3.5), $\gamma_{i}=i$. The first extrapolation is

$$
\mathcal{A}_{\text {inf }, 1}^{\epsilon}=-\mathcal{A}_{\text {inf }}^{\epsilon}+2 \mathcal{A}_{\text {inf }}^{\epsilon / 2}
$$

which is a second-order approximation of $\mathcal{A}_{\text {inf }}$, and the second extrapolation is

$$
\mathcal{A}_{i n f, 2}^{\epsilon}=\frac{2^{2} \mathcal{A}_{i n f, 1}^{\epsilon / 2}-\mathcal{A}_{i n f, 1}^{\epsilon}}{2^{2}-1}=\mathcal{A}_{i n f}^{\epsilon} / 3-2 \mathcal{A}_{i n f}^{\epsilon / 2}+8 \mathcal{A}_{\text {inf }}^{\epsilon / 4} / 3
$$

which is a third-order approximation of $\mathcal{A}_{\text {inf }}$. 


\section{REFERENCES}

[1] G. Bastard, Wave Mechanics Applied to Semiconductor Heterostructures, les éditions de physique, Les Ulis Cedex, France, 1988.

[2] M. Ehrhardt and C. Zheng, Exact artificial boundary conditions for problems with periodic structures, J. Comput. Phys., 227, 6877-6894, 2008.

[3] M. Ehrhardt, H. Han and C. Zheng, Numerical simulation of waves in periodic structures, Commun. Comput. Phys., 5(5), 849-872, 2009.

[4] S. Fliss and P. Joly, Exact boundary conditions for time-harmonic wave propagation in locally perturbed periodic media, Appl. Numer. Math, 2008 (Special Issue of WONAPDE 2007: The Second Chilean Workshop on Numerical Analysis of Partial Differential Equations, Concepión, Chile, Jan 16-19, 2007).

[5] S.P. Guo and S. Albin, Numerical techniques for excitation and analysis of defect modes in photonic crystals, Opt. Express, 11, 1080-1089, 2003.

[6] Z. Han, E. Forsberg and S. He, Surface plasmon Bragg gratings formed in metal-insulator-metal waveguides, IEEE Photonics Techn. Lett., 19, 91-93, 2007.

[7] L. Hörmander, Linear Partial Differential Operators, Grundlehren der mathematischen Wissenschaften, Springer-Verlag, 116, 1969.

[8] R. Leis, Initial boundary value problems in mathematical physics, Stuttgart, B.G. Teubner, Chichester, New York, Wiley, 1986.

[9] J.D. Joannopoulos, S.G. Johnson, J.N. Winn and R.D. Meade, Photonic Crystals: Molding the Flow of Light, second edition, Princeton University Press, 2008.

[10] P. Joly, J.R. Li and S. Fliss, Exact boundary conditions for periodic waveguides containing a local perturbation, Commun. Comput. Phys., 1, 945-973, 2006.

[11] P. Kuchment, Floquet theory for partial differential equations, Operator Theory: Advances and Applications, Birkhäuser Verlag, Basel, 60, 1993.

[12] P. Kuchment, The mathematics of photonic crystals, Mathematical Modeling in Optical Science, Frontiers in Applied Mathematics, SIAM, Philadelphia, 7, 22, 2001.

[13] C.B. Moler and G.W. Stewart, An algorithm for generalized matrix eigenvalue problems, SIAM J. Numer. Anal., 10(2), 241-256, 1973.

[14] K. Morgenröther, On the principles of limiting absorption and limit amplitude for a class of locally perturbed waveguides. Part 1: time-independent theory, Math. Methods Appl. Sci., 10, 125-144, 1988.

[15] C. Potel, Ph. Gatignol and J.F. De Belleval, Energetic criterion for the radiation of floquet waves in infinite anisotropic periodically multilayered media, Acustica-Acta Acustica, 87, 340-351, 2001.

[16] K. Sakoda, Optical Properties of Photonic Crystals, Springer-Verlag, Berlin, 2001.

[17] D.R. Smith, J.B. Pendry and M.C.K. Wiltshire, Metamaterials and negative refractive index, Science, 305, 788-792, 2004.

[18] T. Søndergard, S.I. Bozhevolnyi and A. Boltasseva, Theoretical analysis of ridge gratings for long-range surface plasmon polaritons, Phys. Rev. B, 73, 045320, 2006.

[19] J. Stoer and R. Bulirsch, Introduction to Numerical Analysis, R. Bartels, W. Gautschi and C. Witzgall (translated), New York, Springer-Verlag, 1991.

[20] J. Sun and C. Zheng, Numerical scattering analysis of TE plane waves by a metallic diffraction grating with local defects, J. Opt. Soc. Am. A, 26(1), 156-162, 2009.

[21] F. Tisseur and K. Meerbergen, The quadratic eigenvalue problem, SIAM Rev., 43(2), 235-286, 2001.

[22] B.R. Vainberg, Principles of radiation, limit absorption and limit amplitude in the general theory of partial differential equations, Russ. Math. Surv., 21, 115-193, 1966.

[23] A. Wacker, Semiconductor Superlattices: a model system for nonlinear transport, Phys. Rep., 357, 1-111, 2002.

[24] L. Yuan and Y.Y. Lu, A recursive doubling Dirichlet-to-Neumann map method for periodic waveguides, J. Lightwave Technology, 25, 3649-3656, 2007.

[25] C. Zheng, An exact boundary condition for the Schrödinger equation with sinusoidal potentials at infinity, Commun. Comput. Phys., 3, 641-658, 2007. 